\title{
I-Pre-Cauchy Double Sequences and Orlicz Functions
}

\author{
Vakeel A. Khan ${ }^{1}$, Nazneen Khan ${ }^{1}$, Ayhan Esi $^{2}$, Sabiha Tabassum ${ }^{3}$ \\ ${ }^{1}$ Department of Mathematics, Aligarh Muslim University, Aligarh, India \\ ${ }^{2}$ Department of Mathematics, Science and Art Faculty, Adiyaman University, Adiyaman, Turkey \\ ${ }^{3}$ Department of Applied Mathematics, Zakir Hussain College of Engineering and Technology, \\ Aligarh Muslim University, Aligarh, India \\ Email: vakhanmaths@gmail.com,nazneen4maths@gmail.com, aesi23@hotmail.com
}

Received March 1, 2013; revised April 3, 2013; accepted April 12, 2013

Copyright (c) 2013 Vakeel A. Khan et al. This is an open access article distributed under the Creative Commons Attribution License, which permits unrestricted use, distribution, and reproduction in any medium, provided the original work is properly cited.

\section{ABSTRACT}

Let $x=\left(x_{i j}\right)$ be a double sequence and let $M$ be a bounded Orlicz function. We prove that $x$ is I-pre-Cauchy if and only if $I-\lim _{m n} \frac{1}{m^{2} n^{2}} \sum_{i, p \leq m} \sum_{j, q \leq n} M\left(\frac{\left|x_{i j}-x_{p q}\right|}{\rho}\right)=0$. This implies a theorem due to Connor, Fridy and Klin [1], and Vakeel A. Khan and Q. M. Danish Lohani [2].

Keywords: Ideal; Filter; Paranorm; I-Convergent; Invariant Mean; Monotone and Solid Space

\section{Introduction}

The concept of statistical convergence was first defined by Steinhaus [3] at a conference held at Wroclaw University, Poland in 1949 and also independently by Fast [4], Buck [5] and Schoenberg [6] for real and complex sequences. Further this concept was studied by Salat [7], Fridy [8], Connor [9] and many others. Statistical convergence is a generalization of the usual notation of convergence that parallels the usual theory of convergence.

A sequence $x=\left(x_{i}\right)$ is said to be statistically convergent to $L$ if for a given $\varepsilon>0$

$$
\lim _{k} \frac{1}{k}\left|\left\{i:\left|x_{i}-L\right| \geq \varepsilon, i \leq k\right\}\right|=0 .
$$

A sequence $x=\left(x_{i}\right)$ is said to be statistically precauchy if

$$
\lim _{k} \frac{1}{k^{2}}\left|\left\{(j, i):\left|x_{i}-x_{j}\right| \geq \varepsilon, j, i \leq k\right\}\right|=0 .
$$

Connor, Fridy and Klin [1] proved that statistically convergent sequences are statistically pre-cauchy and any bounded statistically pre-cauchy sequence with a nowhere dense set of limit points is statistically convergent. They also gave an example showing statistically pre-cauchy sequences are not necessarily statistically convergent (see [10]).

Throughout a double sequence is denoted by $x=\left(x_{i j}\right)$. A double sequence is a double infinite array of elements $x_{i j} \in \mathbb{R}$ for all $i, j \in \mathbb{N}$.

The initial works on double sequences is found in Bromwich [11], Tripathy [12], Basarir and Solancan [13] and many others.

Definition 1.1. A double sequence $\left(x_{i j}\right)$ is called statistically convergent to $L$ if

$$
\lim _{m, n \rightarrow \infty} \frac{1}{m n}|(i, j):| x_{i j}-L|\geq \varepsilon, i \leq m, j \leq n|=0,
$$

where the vertical bars indicate the number of elements in the set.

Definition 1.2. A double sequence $\left(x_{i j}\right)$ is called statistically pre-cauchy if for every $\varepsilon>0$ there exist $p=p(\varepsilon)$ and $q=q(\varepsilon)$ such that

$$
\lim _{m, n \rightarrow \infty} \frac{1}{m^{2} n^{2}}(i, j):\left|x_{i j}-x_{p q}\right| \geq \varepsilon, i \leq m, j \leq n \mid=0 .
$$

Definition 1.3. An Orlicz Function is a function $M:[0, \infty) \rightarrow[0, \infty)$ which is continuous, nondecreasing and convex with $M(0)=0, M(x)>0$ for $x>0$ and $M(x) \rightarrow \infty$, as $x \rightarrow \infty$.

If convexity of $M$ is replaced by $M(x+y) \leq M(x)+M(y)$, then it is called a Modulus function (see Maddox [14]). An Orlicz function may be bounded or unbounded. For example,

$M(x)=x^{p}(0<p \leq 1)$ is unbounded and $M(x)=\frac{x}{x+1}$ 
is bounded (see Maddox [14]).

Lindenstrauss and Tzafriri [15] used the idea of Orlicz functions to construct the sequence space,

$$
\ell_{M}=\left\{x \in \omega: \sum_{k=1}^{\infty} M\left(\frac{\left|x_{k}\right|}{\rho}\right)<\infty, \text { for some } \rho>0\right\} .
$$

The space $\ell_{M}$ is a Banach space with the norm

$$
\|x\|=\inf \left\{\rho>0: \sum_{k=1}^{\infty} M\left(\frac{\left|x_{k}\right|}{\rho}\right) \leq 1\right\}
$$

The space $\ell_{M}$ is closely related to the space $\ell_{p}$ which is an Orlicz sequence space with $M(x)=x^{p}$ for $1 \leq p<\infty$.

An Orlicz function $M$ is said to satisfy $\Delta_{2}$ condition for all values of $x$ if there exists a constant $K>0$ such that $M(L x) \leq K L M(x)$ for all values of $L>1$.

The study of Orlicz sequence spaces have been made recently by various authors [1,2,16-20]).

In [1], Connor,Fridy and Klin proved that a bounded sequence $x=\left(x_{k}\right)$ is statistically pre-cauchy if and only if

$$
\lim _{k} \frac{1}{k^{2}} \sum_{i, j \leq k}\left(\left|x_{i}-x_{j}\right|\right)=0 .
$$

The notion of I-convergence is a generalization of statistical convergence. At the initial stage it was studied by Kostyrko, Salat, Wilezynski [21]. Later on it was studied by Salat, Tripathy, Ziman [22] and Demirci [23], Tripathy and Hazarika [24-26]. Here we give some preliminaries about the notion of I-convergence.

Definition 1.4. [20,27] Let $X$ be a non empty set. Then a family of sets $I \subseteq 2^{X}$ ( $2^{X}$ denoting the power set of $X$ ) is said to be an ideal in $X$ if

(i) $\varnothing \in I$

(ii) $I$ is additive i.e $A, B \in I \Rightarrow A \cap B \in I$.

(iii) $I$ is hereditary i.e $A \in I, B \subseteq A \Rightarrow B \in I$.

An Ideal $I \subseteq 2^{X}$ is called non-trivial if $I \neq 2^{X}$. A non-trivial ideal $I \subseteq 2^{X}$ is called admissible if $\{\{x\}: x \in X\} \subseteq I$.

A non-trivial ideal $I$ is maximal if there cannot exist any non-trivial ideal $J \neq I$ containing $I$ as a subset.

For each ideal $I$, there is a filter $£(I)$ corresponding to I. i.e.

$$
£(I)=\left\{K \subseteq N: K^{c} \in I\right\},
$$

where $K^{c}=N-K$.

Definition 1.5. [10,21,28] A double sequence $\left(x_{i j}\right) \in \omega$ is said to be I-convergent to a number $L$ if for every $\epsilon>0$,

$$
\left\{i, j \in \mathbb{N}:\left|x_{i j}-L\right| \geq \epsilon\right\} \in I .
$$

In this case we write $I-\lim x_{i j}=L$.

Definition 1.6. [21] A non-empty family of sets $£(I) \subseteq 2^{X}$ is said to be filter on $X$ if and only if

(i) $\Phi \notin £(I)$,

(ii) For $A, B \in £(I)$ we have $A \cap B \in £(I)$

(iii) For each $A \in £(I)$ and $A \subseteq B$ implies $B \in £(I)$.

\section{Main Results}

In this article we establish the criterion for any arbitrary double sequence to be I-pre-cauchy.

Theorem 2.1. Let $x=\left(x_{i j}\right)$ be a double sequence and let $M$ be a bounded Orlicz function then $x$ is I-preCauchy if and only if

$$
I-\lim _{m n} \frac{1}{m^{2} n^{2}} \sum_{i, p \leq m} \sum_{j, q \leq n} M\left(\frac{\left|x_{i j}-x_{p q}\right|}{\rho}\right)=0 \text {, for some } \rho>0 \text {. }
$$

Proof: Suppose that

$I-\lim _{m n} \frac{1}{m^{2} n^{2}} \sum_{i, p \leq m} \sum_{j, q \leq n} M\left(\frac{\left|x_{i j}-x_{p q}\right|}{\rho}\right)=0$, for some $\rho>0$.

For each $\varepsilon>0, \rho>0$ and $m, n \in I N$ we have that

$A_{1}$

$$
\begin{aligned}
& =\left\{m, n \in I N: M\left(\frac{\left|x_{i j}-x_{p q}\right|}{\rho}\right) \geq \frac{\varepsilon}{2 m n}, i, p \leq m, j, q \leq n\right\} \\
& \in I
\end{aligned}
$$

$A_{1}^{c}$

$$
\begin{aligned}
& =\left\{m, n \in I N: M\left(\frac{\left|x_{i j}-x_{p q}\right|}{\rho}\right)<\frac{\varepsilon}{2 m n}, i, p \leq m, j, q \leq n\right\} \\
& \in I .
\end{aligned}
$$

$$
\begin{aligned}
& \lim _{m n} \frac{1}{m^{2} n^{2}} \sum_{i, p \leq m} \sum_{j, q \leq n} M\left(\frac{\left|x_{i j}-x_{p q}\right|}{\rho}\right) \\
& =\lim _{m n} \frac{1}{m^{2} n^{2}} \sum_{\left|x_{i j}-x_{p q}\right|<\frac{\varepsilon}{2 m n}} M\left(\frac{\left|x_{i j}-x_{p q}\right|}{\rho}\right) \\
& +\lim _{m n} \frac{1}{m^{2} n^{2}} \sum_{\left|x_{i j}-x_{p q}\right| \geq \frac{\varepsilon}{2 m n}} M\left(\frac{\left|x_{i j}-x_{p q}\right|}{\rho}\right) \\
& \geq \lim _{m n} \frac{1}{m^{2} n^{2}} \sum_{\left|x_{i j}-x_{p q}\right| \geq \frac{\varepsilon}{2 m n}} M\left(\frac{\left|x_{i j}-x_{p q}\right|}{\rho}\right)
\end{aligned}
$$

Now by (1) and (2) we have 


$$
\left\{m, n \in I N: \lim _{m n} \frac{1}{m^{2} n^{2}} \sum_{i, p \leq m} \sum_{j, q \leq n} M\left(\frac{\left|x_{i j}-x_{p q}\right|}{\rho}\right) \geq \varepsilon, i, p \leq m j, q \leq n\right\} \subset A_{1} \cup A_{1}^{c} \in I .
$$

thus $x$ is I-pre-Cauchy.

Now conversely suppose that $x$ is I-pre-Cauchy, and that $\varepsilon$ has been given.

Then we have

$$
\left\{m, n \in I N: \lim _{m n} \frac{1}{m^{2} n^{2}} \sum_{i, p \leq m} \sum_{j, q \leq n} M\left(\frac{\left|x_{i j}-x_{p q}\right|}{\rho}\right) \geq \varepsilon, i, p \leq m \quad j, q \leq n\right\} \subset A_{1} \cup A_{1}^{c} \in I .
$$

where,

$$
\begin{aligned}
& =\left\{m, n \in I N: M\left(\frac{\left|x_{i j}-x_{p q}\right|}{\rho}\right) \geq \frac{\varepsilon}{2 m n}, i, p \leq m j, q \leq n\right\} \\
& \in I, \\
& A_{1}^{c} \\
& =\left\{m, n \in I N: M\left(\frac{\left|x_{i j}-x_{p q}\right|}{\rho}\right)<\frac{\varepsilon}{2 m n}, i, p \leq m j, q \leq n\right\} \\
& \in I .
\end{aligned}
$$

Let $\delta>0$ be such that $M(\delta)<\frac{\varepsilon}{2}$. Since $M$ is a bounded Orlicz function there exists an integer $B$ such that $M(x)<\frac{B}{2}$ for all $x \geq 0$. Therefore, for each $m, n \in I N$,

$$
\begin{aligned}
& \lim _{m n} \frac{1}{m^{2} n^{2}} \sum_{i, p \leq m} \sum_{j, q \leq n} M\left(\frac{\left|x_{i j}-x_{p q}\right|}{\rho}\right) \\
& =\lim _{m n} \frac{1}{m^{2} n^{2}} \sum_{\left|x_{i j}-x_{p q}\right|<\frac{\varepsilon}{2 m n}} M\left(\frac{\left|x_{i j}-x_{p q}\right|}{\rho}\right) \\
& +\lim _{m n} \frac{1}{m^{2} n^{2}} \sum_{\left|x_{i j}-x_{p q}\right|} M \frac{\varepsilon}{2 m n}\left(\frac{\left|x_{i j}-x_{p q}\right|}{\rho}\right) \\
& \leq M(\delta)+\lim _{m, n} \frac{1}{m^{2} n^{2}} \sum_{i, p \leq m} \sum_{j, q \leq n} M\left(\frac{\left|x_{i j}-x_{p q}\right|}{\rho}\right) \\
& \leq \frac{\varepsilon}{2}+\frac{B}{2}\left(\frac{1}{m^{2} n^{2}}\left|\left\{(i, j):\left|x_{i j}-x_{p q}\right| \geq \varepsilon, i, p \leq m, j, q \leq n\right\}\right|\right) \\
& \leq \varepsilon+B\left(\frac{1}{m^{2} n^{2}}\left|\left\{(i, j):\left|x_{i j}-x_{p q}\right| \geq \varepsilon, i, p \leq m, j, q \leq n\right\}\right|\right)
\end{aligned}
$$

Since $x$ is I-pre-Cauchy, there is an IN such that the right hand side of (3) is less than $\varepsilon$ for all $m, n \in I N$. Hence

$$
I-\lim _{m, n} \frac{1}{m^{2} n^{2}} \sum_{i, p \leq m} \sum_{j, q \leq n} M\left(\frac{\left|x_{i j}-x_{p q}\right|}{\rho}\right)=0 .
$$

Theorem 2.2. Let $x=\left(x_{i j}\right)$ be a double sequence and let $M$ be a bounded Orlicz function then $x$ is I-convergent to $L$ if and only if

$$
I-\lim _{m, n} \frac{1}{m n} \sum_{i=1}^{m} \sum_{j=1}^{n} M\left(\frac{\left|x_{i j}-L\right|}{\rho}\right)=0 \text {, for some } \rho>0 .
$$

Proof: Suppose that

$$
I-\lim _{m, n} \frac{1}{m n} \sum_{i=1}^{m} \sum_{j=1}^{n} M\left(\frac{\left|x_{i j}-L\right|}{\rho}\right)=0 \text {, for some } \rho>0 .
$$

with an Orlicz function $M$, then $x$ is I-convergent to $L$ (See [1])

Conversely suppose that $x$ is I-convergent to $L$. We can prove this in similar manner as in Theorem 2.1 assuming that

$$
I-\lim _{m, n} \frac{1}{m n} \sum_{i=1}^{m} \sum_{j=1}^{n} M\left(\frac{\left|x_{i j}-L\right|}{\rho}\right)=0 \text {, for some } \rho>0 .
$$

and $M$ being a bounded Orlicz function.

Corollary 2.3. A sequence $x=\left(x_{i j}\right)$ is I-convergent if and only if

$$
I-\lim _{m, n} \frac{1}{m^{2} n^{2}} \sum_{i, p \leq m} \sum_{j, q \leq n}\left|x_{i j}-x_{p q}\right|=0 .
$$

Proof: Let $M(x)=x$. Then

$$
M\left(\frac{\left|x_{i j}-x_{p q}\right|}{\rho}\right) \leq\left|x_{i j}-x_{p q}\right|
$$

for all $i, p \leq m, j, q \leq n$ and for $m, n \in I N$

Let

$B_{1}$

$$
=\left\{m, n \in I N: M\left(\frac{\left|x_{i j}-x_{p q}\right|}{\rho}\right)<\varepsilon, i, p \leq m, j, q \leq n\right\}
$$


and

$$
\begin{aligned}
& B_{1}^{c} \\
& =\left\{m, n \in I N: M\left(\frac{\left|x_{i j}-x_{p q}\right|}{\rho}\right) \geq \varepsilon, i, p \leq m, j, q \leq n\right\} \\
& \in I .
\end{aligned}
$$

Therefore from (4) and (5) we have,

$$
\begin{aligned}
& \left\{m, n \in I N: M\left(\frac{\left|x_{i j}-x_{p q}\right|}{\rho}\right) \geq \varepsilon, i, p \leq m, j, q \leq n\right\} \\
& \subset B_{1} \cup B_{1}^{c} \in I .
\end{aligned}
$$

Hence

$$
I-\lim _{m, n} \frac{1}{m^{2} n^{2}} \sum_{i, p \leq m} \sum_{j, q \leq n}\left|x_{i j}-x_{p q}\right|=0 .
$$

if and only if

$$
I-\lim _{m, n} \frac{1}{m^{2} n^{2}} \sum_{i, p \leq m} \sum_{j, q \leq n} M\left(\frac{\left|x_{i j}-x_{p q}\right|}{\rho}\right)=0 .
$$

By an immediate application of Theorem 2.1 we get the desired result.

Corollary 2.4. A sequence $x=\left(x_{i j}\right)$ is I-convergent to $L$ if and only if

$$
I-\lim _{m, n} \frac{1}{m n} \sum_{i=1}^{m} \sum_{j=1}^{n}\left|x_{i j}-L\right|=0
$$

Proof: Let $M(x)=x$.

We can prove this in the similar manner as in the proof of Corollary 2.3.

\section{Acknowledgements}

The authors would like to record their gratitude to the reviewer for his careful reading and making some useful corrections which improved the presentation of the paper.

\section{REFERENCES}

[1] J. Connor, J. A. Fridy and J. Kline, "Statistically PreCauchy Sequence,” Analysis, Vol. 14, 1994, pp. 311-317.

[2] A. K. Vakeel and Q. M. Danish Lohani, "Statistically Pre-Cauchy Sequences and Orlicz Functions," Southeast Asian Bulletin of Mathematics, Vol. 31, No. 6, 2007, pp. 1107-1112.

[3] H. Steinhaus, "Sur la Convergence Ordinaire et la Convergence Asymptotique,” Colloquium Mathematicum, Vol. 2, 1951, pp. 73-74.

[4] H. Fast, "Sur la Convergence Statistique,” Colloquium Mathematicum, Vol. 2, 1951, pp. 241-244.

[5] R. C. Buck, “Generalized Asymptotic Density,” American Journal of Mathematics, Vol. 75, No. 2, 1953, pp. 335346.
[6] I. J. Schoenberg, "The Integrability of Certain Functions and Related Summability Methods,” The American Mathematical Monthly, Vol. 66, 1959, pp. 361-375.

[7] T. Salat, "On Statistically Convergent Sequences of Real Numbers,” Mathematica Slovaca, Vol. 30, 1980, pp. 139150.

[8] J. A. Fridy, “On Statistical Convergence,” Analysis, Vol 5, 1985, pp. 301-311.

[9] J. S. Connor, "The Statistical and Strong P-Cesaro Convergence of Sequences,” Analysis, Vol. 8, 1988, pp. 4763.

[10] M. Gurdal, "Statistically Pre-Cauchy Sequences and Bounded Moduli," Acta et Commentationes Universitatis Tarytensis de Mathematica, Vol. 7, 2003, pp. 3-7.

[11] T. J. I. Bromwich, "An Introduction to the Theory of Infinite Series,” MacMillan and Co. Ltd., New York, 1965.

[12] B. C. Tripathy, "Statistically Convergent Double Sequences,” Tamkang Journal of Mathematics, Vol. 32, No. 2, 2006, pp. 211-221.

[13] M. Basarir and O. Solancan, "On Some Double Seuence Spaces," The Journal of The Indian Academy of Mathematics, Vol. 21, No. 2, 1999, pp. 193-200.

[14] I. J. Maddox, "Elements of Functional Analysis," Cambridge University Press, Cambridge, Cambridge, 1970.

[15] J. Lindenstrauss and L. Tzafriri, "On Orlicz Sequence Spaces,” Israel Journal of Mathematics, Vol. 10, No. 3, 1971, pp. 379-390. doi:10.1007/BF02771656

[16] M. Et, “On Some New Orlicz Sequence Spaces,” Journal of Analysis, Vol. 9, 2001, pp. 21-28.

[17] S. D. Parashar and B. Choudhary, "Sequence Spaces Defined by Orlicz Function,” Indian Journal of Pure and Applied Mathematics, Vol. 25, 1994, pp. 419-428.

[18] B. C. Tripathy and Mahantas, "On a Class of Sequences Related to the $\mathrm{l}^{\mathrm{P}}$ Space Defined by the Orlicz Functions," Soochow Journal of Mathematics, Vol. 29, No. 4, 2003, pp. 379-391.

[19] A. K. Vakeel and S. Tabassum, "Statistically Pre-Cauchy Double Sequences and Orlicz Functions," Southeast Asian Bulletin of Mathematics, Vol. 36, No. 2, 2012, pp. 249-254.

[20] A. K. Vakeel, K. Ebadullah and A Ahmad, "I-Pre-Cauchy Sequences and Orlicz Functions," Journal of Mathematical Analysis, Vol. 3, No. 1, 2012, pp. 21-26.

[21] P. Kostyrko, T. Salat and W. Wilczynski, "I-Convergence,” Real Analysis Exchange, Vol. 26, No. 2, 2000, pp. 669-686.

[22] T. Salat, B. C. Tripathy and M. Ziman, "On Some Properties of I-Convergence,” Tatra Mountains Mathematical Publications, Vol. 28, 2004, pp. 279-286.

[23] K. Demirci, “I-Limit Superior and Limit Inferior," Mathematical Communications, Vol. 6, 2001, pp. 165-172.

[24] B. C. Tripathy and B. Hazarika, "Paranorm I-Convergent Sequence Spaces,” Mathematica Slovaca, Vol. 59, No. 4, 2009, pp. 485-494. doi:10.2478/s12175-009-0141-4

[25] B. C. Tripathy and B. Hazarika, "Some I-Convergent Se- 
quence Spaces Defined by Orlicz Function,” Acta Mathematica Applicatae Sinica, Vol. 27, No. 1, 2011, pp. 149154. doi:10.1007/s10255-011-0048-Z

[26] B. C. Tripathy and B. Hazarika, "I-Monotonic and I-Convergent Sequences,” Kyungpook Mathematical Journal, Vol. 51, No. 2, 2011, pp. 233-239.

doi:10.5666/KMJ.2011.51.2.233
[27] A. K. Vakeel, K. Ebadullah and S. Suthep, "On a New I-Convergent Sequence Spaces,” Analysis, Vol. 32, No. 3, 2012, pp. 199-208. doi:10.1524/anly.2012.1148

[28] M. Gurdal and M. B. Huban, “On I-Convergence of Double Sequences in the Topology induced by Random 2Norms," Matematicki Vesnik, Vol. 65, No. 3, 2013, pp. 1-13. 